\title{
BMC Biotechnology reviewer acknowledgement 2015
}

Dafne Solera

\section{Contributing reviewers}

The editors of BMC Biotechnology would like to thank all our reviewers who have contributed to the journal in Volume 15 (2015).

\author{
Sirajunnisa Abdul Razack \\ India
}

Sabu Abdulhameed
India

Robert Abramovitch

USA

Warish Ahmed

Australia

Christian Ahrens

Switzerland

Hal Alper

USA

T. R. Anish

USA

Wataru Aoi

Japan

Klaus-J. Appenroth

Germany

Ana C. M. Arisi

Brazil

Ranjana Arya

India

Stéphane Aymerich

France

Jongyoun Baik

USA

\author{
Pedro Baptista \\ Spain \\ Neil Barclay \\ UK \\ Karl Bayer \\ Austria \\ Frank Bedon \\ Australia
}

Evguenia Bekman

Portugal

Martina Bellasio

Austria

Harry Beller

USA

Isabelle Benoit

Netherland

Aleš Berlec

Slovenia

Maurizio Bettiga

Sweden

Silvia Bidarra

Portugal

Robert Bischof

Austria

Pernilla Bjerling

Sweden
John Blackwood

UK

Lars Mathias Blank

Germany

Andreas Bommarius

USA

Joseph Boothe

Canada

Massimiliano Borgogna

Italy

Nicole Borth

Austria

José Bragança

Portugal

David Brindley

Singapore

Mike Brisco

Australia

Jochen Büchs

Germany

Andreas Buehlmann

Switzerland

Matthias Bureik

China

Thomas Burmeister

Germany

Correspondence: Dafne.solera@biomedcentral.com

BioMed Central, Floor 6, 236 Gray's Inn Road, London WC1X 8HB, UK 
Ornella Calderini

Italy

Pinar Calik

Turkey

Andrea Camattari

Singapore

Jijuan Cao

China

Hyung Joon Cha

South Korea

Mao Xiang Chen

UK

Rumei Chen

China

Tomohiro Chiba

Japan

Bijan Choudhury

India

Se Chul Chun

South Korea

Donatella Cimini

Italy

Dennis Claessen

Netherland

\section{Martin Clynes \\ Ireland}

Uli Commandeur

Germany

Tony Conner

New Zealand

Olga Correa

Argentina

Casey Crooks

USA

Jian Dong Cui

China

David Culley

USA

Tamás Dalmay

UK

Paul Daly

UK

Pascale Daran-Lapujade

Netherland
Mark Davis

USA

Noushin Davoudi

Iran

Liziane Maria De Lima

Brazil

Donatella De Pascale

Italy

Sandra De Weert

Netherland

Tigst Demeke

Canada

Vipin Deo

Japan

Leo Despaux

USA

Luigi Di Costanzo

USA

Isabel Dias

Portugal

Adiphol Dilokpimol

Netherland

Dino Dissel

Netherland

Jinhua Dong

Japan

Giovanna Donnarumma

Italy

Sarah E. F. D'Orazio

USA

Martin Dragosits

Austria

Dion Du Plessis

South Africa

Nishant Dwivedi

USA

Colin Eady

New Zealand

Brigitta Ebert

Germany

Lothar Eggeling

Germany

Abdellatif El M'Selmi

France
Dominic Esposito

USA

María Julia Estrella

Argentina

Thaddeus Ezeji

USA

Fan Fan

USA

Zhiliang Fan

USA

Phillip Farabaugh

USA

Jeanni Fehrsen

South Africa

Hugo Fernandes

Portugal

Tiago Fernandes

Portugal

John J. Finer

USA

Ayelet Fishman

Israel

Hans-Lothar Fuchsbauer Germany

Manuel Fuentes

Spain

Alexander Fulton

Germany

Brigitte Gasser

Austria

Elena Geiser

Germany

Yoram Gerchman

Israel

Aron Geurts

USA

Nasser Ghaemi

Iran

M. Ghosh

India

Paula Giardina

Italy

Rajanish Giri

India 


\begin{tabular}{|c|c|c|}
\hline $\begin{array}{l}\text { Graciela Glikmann } \\
\text { Argentina }\end{array}$ & $\begin{array}{l}\text { Masahito Ikawa } \\
\text { Japan }\end{array}$ & $\begin{array}{l}\text { Hyun Woo Kim } \\
\text { South Korea }\end{array}$ \\
\hline $\begin{array}{l}\text { Ee-Been Goh } \\
\text { USA }\end{array}$ & $\begin{array}{l}\text { Masashi Iwanaga } \\
\text { Japan }\end{array}$ & $\begin{array}{l}\text { Jong-Won Kim } \\
\text { South Korea }\end{array}$ \\
\hline $\begin{array}{l}\text { Billie Gould } \\
\text { Canada }\end{array}$ & $\begin{array}{l}\text { Grzegorz Janusz } \\
\text { Poland }\end{array}$ & $\begin{array}{l}\text { Sang Jick Kim } \\
\text { South Korea }\end{array}$ \\
\hline $\begin{array}{l}\text { Catherine Grgicak } \\
\text { USA }\end{array}$ & $\begin{array}{l}\text { Clark Jeffries } \\
\text { USA }\end{array}$ & $\begin{array}{l}\text { Yeon-Gu Kim } \\
\text { South Korea }\end{array}$ \\
\hline $\begin{array}{l}\text { Ewa Grzebelus } \\
\text { Poland }\end{array}$ & $\begin{array}{l}\text { Jingfang Ju } \\
\text { USA }\end{array}$ & $\begin{array}{l}\text { Stephan Kirchmaier } \\
\text { Germany }\end{array}$ \\
\hline $\begin{array}{l}\text { Rani Gupta } \\
\text { India }\end{array}$ & $\begin{array}{l}\text { Yi-Hsu Ju } \\
\text { Taiwan }\end{array}$ & $\begin{array}{l}\text { Heath Klock } \\
\text { USA }\end{array}$ \\
\hline $\begin{array}{l}\text { Taekwang Ha } \\
\text { Denmark }\end{array}$ & $\begin{array}{l}\text { Vipin Kalia } \\
\text { India }\end{array}$ & $\begin{array}{l}\text { Tie Koide } \\
\text { Brazil }\end{array}$ \\
\hline $\begin{array}{l}\text { Henning Hansen } \\
\text { Denmark }\end{array}$ & $\begin{array}{l}\text { Héla Kallel } \\
\text { Tunisia }\end{array}$ & $\begin{array}{l}\text { Jens Kossmann } \\
\text { South Africa }\end{array}$ \\
\hline $\begin{array}{l}\text { Kim Hebelstrup } \\
\text { Denmark }\end{array}$ & $\begin{array}{l}\text { Masamichi Kamihira } \\
\text { Japan }\end{array}$ & $\begin{array}{l}\text { Mauri Kostiainen } \\
\text { Finland }\end{array}$ \\
\hline $\begin{array}{l}\text { Êlke Heine-Dobbernack } \\
\text { Germany }\end{array}$ & $\begin{array}{l}\text { Angelos K. Kanellis } \\
\text { Greece }\end{array}$ & $\begin{array}{l}\text { Harald Kranz } \\
\text { Germany }\end{array}$ \\
\hline $\begin{array}{l}\text { Olivier Henry } \\
\text { Canada }\end{array}$ & $\begin{array}{l}\text { Han Chang Kang } \\
\text { USA }\end{array}$ & $\begin{array}{l}\text { László Kredics } \\
\text { Hungary }\end{array}$ \\
\hline $\begin{array}{l}\text { Pablo Hernández } \\
\text { Spain }\end{array}$ & $\begin{array}{l}\text { Sang-Moo Kang } \\
\text { USA }\end{array}$ & $\begin{array}{l}\text { Jonas Kügler } \\
\text { Germany }\end{array}$ \\
\hline $\begin{array}{l}\text { Hirofumi Hirai } \\
\text { Japan }\end{array}$ & $\begin{array}{l}\text { Ajit Kanwal } \\
\text { India }\end{array}$ & $\begin{array}{l}\text { Abhishek Kumar } \\
\text { Germany }\end{array}$ \\
\hline $\begin{array}{l}\text { Caroline Hoemann } \\
\text { Canada }\end{array}$ & $\begin{array}{l}\text { Levente Karaffa } \\
\text { Hungary }\end{array}$ & $\begin{array}{l}\text { Guruprasad Kuntamallappanavar } \\
\text { USA }\end{array}$ \\
\hline $\begin{array}{l}\text { Raimund Hoffrogge } \\
\text { Germany }\end{array}$ & $\begin{array}{l}\text { Mythreye Karthikeyan } \\
\text { USA }\end{array}$ & $\begin{array}{l}\text { Sang-Soo Kwak } \\
\text { South Korea }\end{array}$ \\
\hline $\begin{array}{l}\text { Barbara Hohn } \\
\text { Switzerland }\end{array}$ & $\begin{array}{l}\text { Tatsuya Kato } \\
\text { Japan }\end{array}$ & $\begin{array}{l}\text { Kyu Kwon } \\
\text { South Korea }\end{array}$ \\
\hline $\begin{array}{l}\text { Hiroyuki Honda } \\
\text { Japan }\end{array}$ & $\begin{array}{l}\text { Arvind Kayastha } \\
\text { India }\end{array}$ & $\begin{array}{l}\text { Soonjo Kwon } \\
\text { South Korea }\end{array}$ \\
\hline $\begin{array}{l}\text { Thomas Horbett } \\
\text { USA }\end{array}$ & $\begin{array}{l}\text { Todd Kelley } \\
\text { USA }\end{array}$ & $\begin{array}{l}\text { Pavel Kyslík } \\
\text { Czech Republic }\end{array}$ \\
\hline $\begin{array}{l}\text { David P. Horvath } \\
\text { USA }\end{array}$ & $\begin{array}{l}\text { Nawazi Khan } \\
\text { USA }\end{array}$ & $\begin{array}{l}\text { Rup Lal } \\
\text { India }\end{array}$ \\
\hline $\begin{array}{l}\text { Huang Huang } \\
\text { USA }\end{array}$ & $\begin{array}{l}\text { Chang Sup Kim } \\
\text { South Korea }\end{array}$ & $\begin{array}{l}\text { Marina Laura } \\
\text { Italy }\end{array}$ \\
\hline $\begin{array}{l}\text { Michael Hust } \\
\text { Germany }\end{array}$ & $\begin{array}{l}\text { Haeng-Hoon Kim } \\
\text { South Korea }\end{array}$ & $\begin{array}{l}\text { Denis Leclerc } \\
\text { Canada }\end{array}$ \\
\hline $\begin{array}{l}\text { Taisen Iguchi } \\
\text { Japan }\end{array}$ & $\begin{array}{l}\text { Ho Min Kim } \\
\text { South Korea }\end{array}$ & $\begin{array}{l}\text { Ruifang Li } \\
\text { China }\end{array}$ \\
\hline
\end{tabular}


Jiazhang Lian

USA

Shuli Liang

China

Junita Liebenberg

South Africa

Melissa Lin

Singapore

Ruijie Liu

China

Xiaolin Liu

China

Yajun Liu

China

Zewen Liu

China

Zoachang Liu

China

Verena Lohr

Germany

Liam Longo

Israel

Catia Giovanna Lopresto

Italy

Marina Lotti

Italy

Xiaochu Lou

USA

Arianna Lovato

Italy

Hua Lu

USA

Xiaofeng Lu

USA

Roland Ludwig

Austria

Taina Lundell

Finland

Hong Luo

USA

Yinggang Luo

China

Jill Madine

UK
François Madore

Canada

Ivan G. Maia

Brazil

Giuseppe Manco

Italy

Giuseppe Mandolino

Italy

Maheswaran Mani

India

Ben Mans

South Africa

Francois Maree

South Africa

Helena Maresová

Czech Republic

Celestina Mariani

Netherland

Nelson Marmiroli

Italy

Enzo Martegani

Italy

Dirk E. Martens

Netherland

Ludmila Martinkova

Czech Republic

Albino Martins

Portugal

Autar Mattoo

USA

Steve Maxwell

USA

Mark Mccann

New Zealand

Praveen Mehta

India

Wenzhao Meng

USA

Severine Menoret

France

Joachim Messing

USA

Yungen Miao

China
Michael Miller

USA

Yoshiko Minami

Japan

Nuno Mira

Portugal

Bruno Miroux

France

Narayan Chandra Mishra India

Saroj Mishra

India

Gisele Monteiro

Brazil

Emilio Montesinos

Spain

Kuntal Mukerjee

USA

Ulrike G. Munderloh

USA

Jolanda Munster

UK

Sean Vincent Murphy

USA

Nafiseh Nafissi

Canada

Hiroyuki Nakai

USA

Hiroshi Nakayama

Japan

Kee Woei Ng

Singapore

Jean Marc Nicaud

France

Ken-Ichi Nishijima

Japan

Yuval Nov

Israel

Cory Nykiforuk

Canada

Kevin O'Connor

Ireland

Rosa Marıa Oliart-Ros

Mexico 
Chalermporn Ongvarrasopone

Slovenia

Gary R. Ostroff

USA

Seung Pil Pack

South Korea

Hongbo Pang

USA

Nádia Parachin

Brazil

Sheldon J. Park

USA

Sungjo Park

USA

Ermenegilda Parrilli

Italy

Siavash Partow

Canada

Ashok Patel

India

Basant Patel

India

Leslie Pedrioli

UK

Matthias Peipp

Germany

Yingjie Peng

USA

James Petrie

Australia

Mauro Petrillo

Italy

Jitka Petrlova

Sweden

Rogério Pezato

Brazil

Steven Poelzing

USA

Matt Posewitz

USA

James Preston

USA

Russ Price

USA
Roland Prielhofer

Austria

Holger Puchta

Germany

Qiudeng Que

USA

Marko Radic

USA

Zeljko Radulovic

USA

Qasim Rafiq

UK

Arun Rajkumar

Denmark

Bruce Ramsay

Canada

Gurinderjit Randhawa

India

Nicolas Rasche

Germany

Norman A. Ratcliffe

UK

Girish Ratnaparkhi

India

Ana Filipa Rodrigues

Portugal

Carlos Rodrigues

Portugal

Susana Rodriguez-Couto

Spain

Gertrudis Rojas

Cuba

Antonio Roldao

Portugal

Carlos Romero

USA

Nancy Roosens

Belgium

Stefan Rose-John

Germany

Daniele Rosellini

Italy

Akihide Ryo

Japan
Xavier Saelens

Belgium

Bhaskar Saha

India

Masakiyo Sakaguchi

Japan

Christiane Salgado

Portugal

Ana Marisa Salgueiro

Portugal

Maurilio Sampaolesi

Belgium

Sébastien Sart

Belgium

Chiara Schiraldi

Italy

Lutz Schmitt

Germany

Bernhard Seiboth

Austria

Seung-Oh Seo

USA

Davide Seruggia

Spain

Luisa Seuanes Serafim

Portugal

Frederick Sheedy

Ireland

Hamid Shegarfi

Noeway

Xiaolin Shen

China

Zhicheng Shen

China

Abhay Shendye

USA

Oded Shoseyov

Israel

Irina Simões

UK

Raushan Singh

USA

Yogendra Singh

India 
Gordon Smyth

Australia

Jonathan Snow

USA

Ola Söderberg

Sweden

Ulrich Soltmann

Germany

Oliver Spadiut

Austria

Alagarsamy Srinivasan

USA

Arun Srivastava

USA

Sanjeeva Srivastava

India

Igor Stagljar

Canada

Joseph Stains

USA

Piotr Stefanowicz

Poland

Matthias Steiger

Austria

Antonietta Stellavato

Italy

Liudmila Stepanova

Russian Federation

C. Neal Stewart

USA

Francesca Storici

USA

Gerald Striedner

Austria

Piruthivi Sukumar

India

Liangliang Sun

USA

Kamilla Swiech

Brazil

György Szittya

Hungary
Rouzbeh Taghizadeh

USA

Tatsuya Takemoto

Japan

Ralf Takors

Germany

Irene Tan

Malaysia

Ryouichi Tanaka

Japan

Yong Tang

Canada

Sheng-Ce Tao

China

Yosuke Tashiro

Japan

Christof Taxis

Germany

Alexander Ter Beek

Netherland

Roger Thilmony

USA

Xu Tian

USA

Viktorija Tomic

Slovenia

Belinda Townsend

UK

Yoshiaki Tsuji

USA

Aysel Ugur

Turkey

Jeroen P. Van Dijk

Netherland

Mirinda Van Kleef

South Africa

Petrus Van Zyl

South Africa

Anikó Várnai

Norway

Ajay Vashisht

USA
Vinod Vathipadiekal

USA

Phanindra Velisetty

USA

Hans Visser

Netherland

Bernd Voedisch

Germany

Jianhua Wang

China

Yimeng Wang

USA

Lawrence Wangh

USA

Hajime Watanabe

Japan

Satoko Watanabe

Japan

Stephen Weeks

Belgium

Benedikt Wefer

Germany

Sven Wegerhoff

Germany

Roland Weis

Austria

Steve Whyard

Canada

Andrew Wilber

USA

David Wilson

USA

Somrutai Winichayakul

New Zealand

Gavin Wright

UK

Qiang Wu

China

Lian Xijun

China

Montarop Yamabhai

Thailand 
Kenji Yamamoto

Japan

Boxu Yan

USA
Ming-Te Yang

Taiwan

Kechun Zhang

USA
Zhanying Zhang

Australia

Shoujing Zhao

China 